\title{
Effects of hyperbaric oxygen therapy on NACHT domain-leucine-rich-repeat- and pyrin domain-containing protein 3 inflammasome expression in rats following spinal cord injury
}

\author{
FANG LIANG ${ }^{1,2}$, CHUNSHENG LI $^{3}$, CHUNJIN GAO $^{1,2}$, ZHUO LI $^{1,2}$, JING YANG $^{1,2}$, \\ XUEHUA LIU ${ }^{1,2}$ and YONG WANG ${ }^{1,2}$ \\ ${ }^{1}$ Department of Hyperbaric Oxygen, Beijing Chaoyang Hospital; ${ }^{2}$ Department of Hyperbaric Oxygen, \\ Beijing Fuxing Hospital; ${ }^{3}$ Department of Emergency, Beijing Chaoyang Hospital, \\ Capital Medical University, Beijing 100020, P.R. China
}

Received March 10, 2014; Accepted November 5, 2014

DOI: $10.3892 / \mathrm{mmr} .2015 .3314$

\begin{abstract}
The clinical application of hyperbaric oxygen therapy (HBOT) in spinal cord injury (SCI) has been reported, however the mechanism underlying its therapeutic effects remains to be elucidated. In the present study, SCI was modeled in male Sprague-Dawley rats. A total of 120 rats were randomly divided into four groups: Sham-operated group (SH); sham-operated and hyperbaric oxygen group (SH+HBO); spinal cord injury group (SCI) and spinal cord injury and hyperbaric oxygen treatment group (SCI+HBO). The rats in each group were randomly divided into five smaller groups (12 h, 1, 3, 7 and 14 days after surgery). The mRNA and protein expression levels of NACHT domain-, leucine-rich-repeat- and pyrin domain-containing protein 3 (NALP3) inflammasome, including NALP3, adaptor molecule apoptosis-associated speck-like protein (ASC) and caspase-1 were determined at several time points following injury. The results of the present study demonstrated that HBOT compromised the mRNA and protein expression levels of NALP3, ASC and caspase- 1 in the SCI model rats and HBOT mitigated SCI-induced interleukin $1 \beta$ release in the injured spinal cord tissue. It was concluded that HBOT is an effective approach, which can prevent against spinal cord injury, likely by inactivating NALP3 inflammasome.
\end{abstract}

Correspondence to: Professor Chunsheng Li, Department of Emergency, Beijing Chaoyang Hospital, Capital Medical University, 8 Baijiazhuang Road, Beijing 100020, P.R. China

E-mail: chunshengli60@163.com

Key words: hyperbaric oxygen therapy, spinal cord injury, NACHT domain-, leucine-rich-repeat-, pyrin domain-containing protein 3 inflammasome

\section{Introduction}

Spinal cord injury (SCI) is a devastating disease, which results in the disruption of neural structures and permanent neurological deficits (1-3). SCI leads to primary injury, which damages cord integrity and secondary injury, which causes further tissue damage and eventually leads to cell death (4). Secondary injury occurs minutes following injury and lasts for several weeks (5) due to ischemia, edema, generation of free radicals and oxidative stress (ROS) and inflammatory responses (6). Among these factors, ROS is important in the stage of secondary injury, which causes inflammation and death of the injured nerve cells (7).

The most generally accepted drug to control secondary damage following SCI is methylprednisolone (MP). However, the side effects caused by this drug limit the clinical application of MP (8). A more safe and effective approach in preventing the onset of edema, ischemia and tissue destruction during the stage of secondary injury is urgently required. Hyperbaric oxygen therapy (HBOT) is a clinical strategy, which involves administering $100 \%$ oxygen for a prescribed period of time at a pressure above that of atmospheric pressure at sea level. This strategy has proven to be beneficial for treating acute and chronic SCI, by increasing oxygen tension in the tissue surrounding the wound $(9,10)$. Previous studies regarding the effects of HBOT on SCI have focussed predominantly on improving neurological function and histological scores (11). However, the molecular mechanism underlying the effects of HBOT remain to be elucidated. As a multimeric protein complex that mediates the release of pro-inflammatory interleukin (IL)-1 $\beta$, the NACHT domain-, leucine-rich-repeat- and pyrin domain-containing protein 3 (NALP3) inflammasome has received considerable attention in SCI (12). The inflammasome consists of NALP3, adaptor molecule apoptosis-associated speck-like protein (ASC) and caspase-1. Formation of the inflammasome results in the production of bioactive IL-1 $\beta$ (13), which triggers the release of other inflammatory cytokines and causes important biological effects associated with tissue 
injury. Villegas et al demonstrated that the activation of the NALP3 inflammasome is an important pathway involved in ROS-mediated lung injury (14). Therefore, activation of the NALP3 inflammasome may also be involved in SCI.

The present study investigated the effects of HBOT on the expression of the NALP3 inflammasome and IL-1 $\beta$ following SCI and revealed that HBOT rescued damaged motor functions following SCI and compromised NALP3 inflammasome expression and IL-1 $\beta$ release. These results improve current understanding and assist in further investigations of the molecular mechanisms underlying and application of HBOT in SCI.

\section{Materials and methods}

Animals. Healthy male Sprague-Dawley rats (2-3-months-old, weighing 250-300 g) were housed in pairs for at least 5 days after their arrival to the laboratory. The rats had access to food and water ad libitum and were maintained in a normal $12 \mathrm{~h}$ light/dark cycles in a regulated environment. The present study was approved and performed in accordance with the ethical guidelines of the Committee on the Ethics of Animal Experiments, Capital Medical University (permit no. 2010-D-013; Beijing, China).

Spinal cord injury model. Spinal cord injury was performed aseptically under anesthesia by intraperitoneal injection of $10 \%$ chloral hydrate at a dose of $350 \mathrm{mg} / \mathrm{kg}$. Using the method described by Basso et al (15), the rats were positioned prone on an operating table, the T10 spinous process area was sterilized, followed by a laminectomy, including T10, to expose the spinal cord. The Multicenter Animal Spinal Cord Injury Study impactor was used in order to produce the SCI. The moderate SCI was created by dropping a $10 \mathrm{~g}$ rod from a distance of $25 \mathrm{~mm}$. The characteristics of a successful model included: A wagging tail reflex in the rats, retraction of the lower limbs and flaccid paralysis of the lower extremities. The animals were allowed to recover from the anesthetic and surgical procedures in intensive care. Post-surgery, the bladder was compressed manually by abdominal pressure twice a day until the bladder reflex was restored. Rats were administered with Penicillin G sodium (Zhengdong Taisheng Pharmaceutical Co., Ltd., Shanxi, China) for 3 days.

Experimental groups. A total of 120 rats were randomly divided into four groups: Sham-operated (SH); sham-operated and hyperbaric oxygen treatment $(\mathrm{SH}+\mathrm{HBO})$; spinal cord injury (SCI) and spinal cord injury and hyperbaric oxygen treatment (SCI+HBO). The rats in each group were then randomly divided into five smaller time-specific sub-groups: $12 \mathrm{~h}, 1,3,7,14$ days after surgery. The rats in the $\mathrm{SH}$ group underwent laminectomy without SCI or HBO treatment.

Hyperbaric oxygen treatment. The $\mathrm{SH}+\mathrm{HBO}$ and $\mathrm{SCI}+\mathrm{HBO}$ rats were placed into a custom-made, transparent acrylic plastic pressure chamber (701 Space Research Institute, Beijing, China) immediately following surgery and received 60 min HBOT at 2 atmospheres absolute (ATA) twice per day at $8 \mathrm{~h}$ intervals for the first 3 days and daily thereafter. Compression air was performed at a rate of $1 \mathrm{~kg} / \mathrm{cm}^{2} / \mathrm{min}$ to 2 ATA/100\% oxygen and was maintained for $60 \mathrm{~min}$. The chamber was ventilated with
$100 \%$ oxygen at a rate of $8 \mathrm{l} / \mathrm{min}$. During HBO exposure, the oxygen contents were continuously monitored and maintained at $\geq 95 \%$. To minimize the effects of diurnal variation, all the $\mathrm{HBO}$ exposures began at $8 \mathrm{AM}$ and $4 \mathrm{PM}$. The rats in the $\mathrm{SH}$ and SCI groups were exposed to normobaric air at 1 ATA for $60 \mathrm{~min}$.

Evaluation of motor function and sample collection. The recovery of motor function was evaluated by Basso-Bettie-Bresnahan (BBB) scores using open-field locomotor assessment $12 \mathrm{~h}, 1,3,7$ and 14 days after surgery (15). In an open-field chamber $(120 \times 120 \mathrm{~cm})$, the behavior of the rats was observed for $5 \mathrm{~min}$ by three individuals in a blinded-manner. The scale was designed to reflect progressive motor rating scores and the BBB score was counted based on movement of the joints of the hind limb, weight-bearing capability, coordination and proper gait and tail position.

Following the evaluation of motor function, the animals were sacrificed using chloral hydrate. The spinal segments of the injured sites were removed. Each sample was stored in liquid nitrogen and used for polymerase chain reaction (PCR) and Western blot analysis.

Reverse transcription quantitative PCR (RT-qPCR). The total RNA was extracted from the frozen spinal cord tissues using TRIzol reagent (Invitrogen Life Technologies, Carlsbad, CA, USA) and a BioEasy SYBR Green I Real Time PCR kit (Sangon Biotech, Shanghai, China). The RNA was then reverse transcribed to synthesize cDNA. qPCR was performed using a Line-Gene sequence detector (Applied Biosystems, Inc., Carlsbad, CA, USA). NALP3, ASC, caspase-1 and actin primers were used as listed in Table I. RT-qPCR was performed using a UU-DNA qRT-PCR real-time detection system (Applied Biosystems, Inc.) using SYBR ${ }^{\circledR}$ green I dye detection (ww). The RT-qPCR reaction was incubated at $94^{\circ} \mathrm{C}$ for $30 \mathrm{sec}$, followed by 45 cycles of $94^{\circ} \mathrm{C}$ for $20 \mathrm{sec}$ and $60^{\circ} \mathrm{C}$ for $25 \mathrm{sec}$. The data were analyzed using the detector software (ABI 7500, software 1.3; Applied Biosystems, Inc.). The RT-qPCR products were verified following electrophoresis on $1 \%$ agarose gels and melting curve analysis. The amplified NALP3, ASC, caspase-1 and actin products were 118, 103, 125 and $128 \mathrm{bp}$, respectively. The relative quantification of mRNA expression levels were calculated using the $2^{-\triangle \Delta C T}$ method.

Protein preparation. The spinal cord tissues were frozen in liquid nitrogen and then stored at $-80^{\circ} \mathrm{C}$ prior to analysis. The tissues were homogenized in a sodium dodecyl sulfate (SDS) sample buffer containing a mixture of protease and phosphatase inhibitors (Sigma-Aldrich, St. Louis, MO). The homogenates were centrifuged at $15,000 \mathrm{x}$ g for $10 \mathrm{~min}$ at $4^{\circ} \mathrm{C}$. The supernatants were collected and the protein concentrations were quantified using a protein assay kit (Pierce Biotechnology, Inc., Rockford, IL, USA).

Western blotting. The proteins (20 $\mu \mathrm{g} / \mathrm{sample})$ were separated using $12 \%$ SDS polyacrylamide gel electrophoresis (SDS-PAGE). Following electrophoresis, the proteins were transferred onto polyvinylidene difluoride membranes (Millipore, Billerica, MA, USA), blocked using 5\% nonfat dry milk for 
Table I. Primer sequences.

Primer

NALP3 forward primer

NALP3 reverse primer

ASC forward primer

ASC reverse primer

Caspase-1 forward primer

Caspase-1 reverse primer

GAPDH forward primer

GAPDH reverse primer
Sequence

NALP3, NACHT domain-leucine-rich-repeat and pyrin domain-containing protein 3; ASC, apoptosis-associated speck-like protein.

Table II. Basso-Bettie-Bresnahan score in each group at different time points following spinal cord injury.

\begin{tabular}{lccrr}
\hline Time & SH & SH+HBO & SCI & SCI+HBO \\
\hline $12 \mathrm{~h}$ & $18.72 \pm 6.05$ & $19.45 \pm 7.24$ & $0.27 \pm 0.51^{\mathrm{a}}$ & $0.28 \pm 0.29^{\mathrm{a}}$ \\
1 day & $19.33 \pm 7.29$ & $20.23 \pm 6.35$ & $0.27 \pm 0.31^{\mathrm{a}}$ & $0.30 \pm 0.26^{\mathrm{a}}$ \\
3 days & $20.25 \pm 7.34$ & $20.49 \pm 7.54$ & $1.03 \pm 0.66^{\mathrm{a}}$ & $1.25 \pm 0.75^{\mathrm{a}}$ \\
7 days & $20.61 \pm 7.58$ & $20.55 \pm 7.16$ & $2.56 \pm 1.24^{\mathrm{a}}$ & $5.27 \pm 2.14^{\mathrm{a}, \mathrm{b}}$ \\
14 days & $20.47 \pm 6.81$ & $20.38 \pm 6.88$ & $7.39 \pm 3.08^{\mathrm{a}}$ & $11.35 \pm 4.22^{\mathrm{a}, \mathrm{b}}$ \\
\hline
\end{tabular}

Values are presented as the mean \pm standard deviation $(\mathrm{n}=6) .{ }^{\mathrm{a}} \mathrm{P}<0.01$, SCI and $\mathrm{SCI}+\mathrm{HBO}$ groups compared with $\mathrm{SH}$ and $\mathrm{SH}+\mathrm{HBO}$ groups; ${ }^{\mathrm{b}} \mathrm{P}<0.05, \mathrm{SCI}+\mathrm{HBO}$ groups compared with the SCI groups. SCI, spinal cord injury group; HBO, hyperbaric oxygen treatment; SH, sham-operated.

$1 \mathrm{~h}$ at room temperature in Tris-buffered saline containing 0.1\% Tween 20 (TBS-T; Amresco, Inc., Solon, OH, USA) and incubated overnight at $4^{\circ} \mathrm{C}$ with rat monoclonal anti-NALP3 (1:1000, Santa Cruz Biotechnology, Inc., Santa Cruz, CA, US), anti-ASC (1:1,000, Santa Cruz Biotechnology, Inc.) and anti-caspases-1 (1:2,000, Santa Cruz Biotechnology, Inc.) antibodies. The membranes were washed in TBS-T and were incubated with horseradish peroxidase-labeled secondary antibody (Santa Cruz Biotechnology, Inc.) for 2-3 h at room temperature. Following this, proteins were detected using enhanced chemiluminescence agents (ECL Plus, Pierce Biotechnology, Inc.) and exposure to X-ray film. To quantify protein levels, the X-ray films were scanned using a Minolta scanner (MFC-7470D; Brother Industries, Ltd, Tokyo, Japan) and analyzed using Adobe Photoshop software. The labeling densities were quantified using LabWorks software (UVP, Upland, CA, USA).

Enzyme-linked immunosorbent assay. The levels of IL-1 $\beta$ in the tissue homogenates were measured using enzyme-linked immunosorbent assay (ELISA) kits (MyBioSource, Inc., San Diego, CA, USA). The pre-coated microtiter plate wells were numbered and $100 \mu \mathrm{l}$ of the sample and standard specimens were added to each well. The assays were performed in duplicate. The optical density (OD) was determined using a microplate reader (Multiskan MK3; Thermo Scientific, Waltham, MA, USA) at $450 \mathrm{~nm}$ and a calibration curve was created by plotting the average $\mathrm{OD}$ values of each calibrator on the Y-axis against the concentration value on the X-axis. The best-fit curve was then produced through the points on the graph.

Statistical analysis. Statistical analysis was performed using SPSS 15.0 software (SPSS, Inc., Chicago, IL, USA). All quantitative data were expressed as the mean \pm standard deviation. One-way analysis of variance was used to test the differences in the BBB scores and the expression of NALP3, ASC and caspase-1. Student's t-test was used for certain BBB scores due to an unequal homogeneity of variance. $\mathrm{P}<0.05$ was considered to indicate a statistically significant difference.

\section{Results}

$H B O T$ increases the $B B B$ score of the rats following spinal cord injury. The BBB scores in the SCI and SCI+HBO groups were significantly lower compared with those in the $\mathrm{SH}$ and $\mathrm{SH}+\mathrm{HBO}$ groups $(\mathrm{P}<0.01)$, although gradual recovery was observed in the rats of the $\mathrm{SCI}$ and $\mathrm{SCI}+\mathrm{HBO}$ groups over time, HBOT caused a significant increase in the BBB score on the 7th and 14th days after surgery compared with the SCI group $(\mathrm{P}<0.05)$. No significant difference was observed between the $\mathrm{BBB}$ scores of the $\mathrm{SH}$ and $\mathrm{SH}+\mathrm{HBO}$ groups (P>0.05; Table II).

HBOT suppresses the SCI-induced increase of the NALP3 inflammasome. Western blot analysis and RT-qPCR were used to analyze the expression levels of the NALP3 
Table III. Effect of hyperbaric oxygen therapy on levels of IL-1 $\beta$ in spinal cord tissue following spinal cord injury (n=6).

\begin{tabular}{lcccc}
\hline Time & SH & SH+HBO & SCI & SCI+HBO \\
\hline $12 \mathrm{~h}$ & $80.23 \pm 7.11$ & $77.35 \pm 4.86$ & $107.69 \pm 4.13^{\mathrm{a}}$ & $102.28 \pm 2.87^{\mathrm{a}}$ \\
1 day & $84.98 \pm 6.25$ & $82.38 \pm 3.95$ & $126.44 \pm 3.37^{\mathrm{a}}$ & $117.35 \pm 5.35^{\mathrm{a}}$ \\
3 days & $79.15 \pm 4.22$ & $82.34 \pm 9.04$ & $104.27 \pm 3.85^{\mathrm{a}}$ & $95.44 \pm 4.55^{\mathrm{b}, \mathrm{c}}$ \\
7 days & $83.88 \pm 3.32$ & $80.45 \pm 5.82$ & $97.24 \pm 2.20^{\mathrm{a}}$ & $89.26 \pm 3.77^{\mathrm{a}, \mathrm{c}}$ \\
14 days & $79.19 \pm 6.27$ & $80.41 \pm 3.65$ & $92.16 \pm 5.65^{\mathrm{a}}$ & $89.22 \pm 4.58^{\mathrm{a}}$ \\
\hline
\end{tabular}

Values are presented as the mean \pm standard deviation $(\mathrm{n}=6)$. ${ }^{\mathrm{a}} \mathrm{P}<0.01$ for $\mathrm{SCI}$ and $\mathrm{SCI}+\mathrm{HBO}$ groups versus $\mathrm{SH}$ and $\mathrm{SH}+\mathrm{HBO}$ groups; ${ }^{\mathrm{P}} \mathrm{P}<0.05$ for $\mathrm{SCI}$ and $\mathrm{SCI}+\mathrm{HBO}$ groups versus $\mathrm{SH}$ and $\mathrm{SH}+\mathrm{HBO}$ groups; and ${ }^{\mathrm{C}} \mathrm{P}<0.01$. SCI, spinal cord injury group; HBO, hyperbaric oxygen treatment; $\mathrm{SH}$, sham-operated.

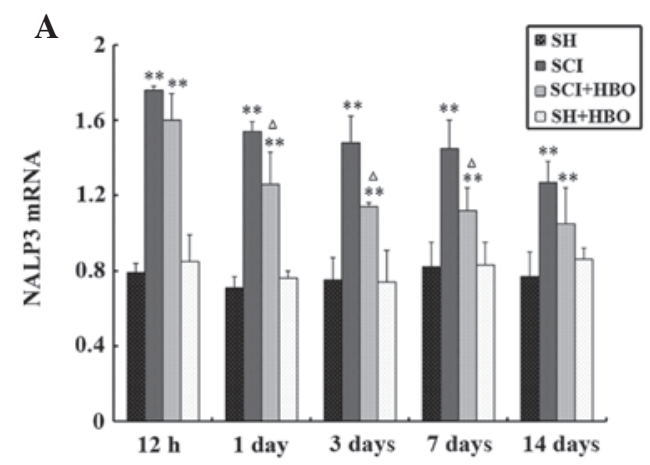

B

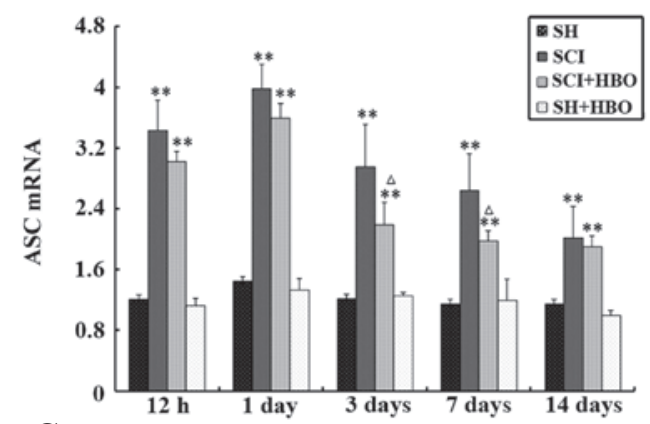

C

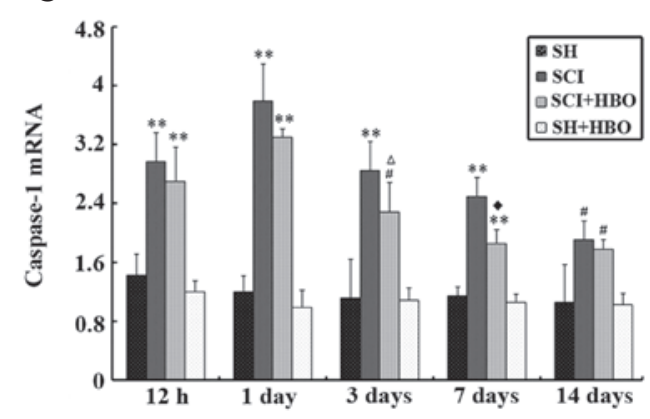

Figure 1. Reverse transcription quantitative polymerase chain reaction analysis of the mRNA level of (A) NALP3, (B) ASC and (C) caspase-1 in the spinal cords of all groups rats at different time points following surgery. Data are presented as the mean \pm standard deviation. ${ }^{* *} \mathrm{P}<0.01 \mathrm{SCI}$ and $\mathrm{SCI}+\mathrm{HBO}$ groups, vs.SH and $\mathrm{SH}+\mathrm{HBO}$ groups; ${ }^{\mathrm{P}}<0.05 \mathrm{SCI}$ and $\mathrm{SCI}+\mathrm{HBO}$ groups, vs. $\mathrm{SH}$ and $\mathrm{SH}+\mathrm{HBO}$ groups; ${ }^{\bullet} \mathrm{P}<0.01$ and ${ }^{\triangle} \mathrm{P}<0.05 \mathrm{SCI}+\mathrm{HBO}$ group, vs. $\mathrm{SCI}$ group. ASC, apoptosis-associated speck-like proteins; SCI, spinal cord injury; $\mathrm{HBO}$, hyperbaric oxygen treatment; $\mathrm{SH}$, sham-operated.

inflammasome, including NALP3, ASC and caspase-1, in the spinal cord tissue. The expression levels of NALP3, ASC and caspase- 1 in the SCI groups were significantly increased compared with those in the $\mathrm{SH}$ and $\mathrm{SH}+\mathrm{HBO}$ groups at all time points $(\mathrm{P}<0.05$ or $\mathrm{P}<0.01$; Figs. $1-4)$. The mRNA and protein expression levels of NALP3 and of ASC and caspase-1 peaked $12 \mathrm{~h}$ and 1 day after surgery, respectively. The mRNA and protein expression levels of NALP3 in the SCI+HBO group were significantly reduced compared with those in the SCI group at 1, 3 and 7 days after surgery $(\mathrm{P}<0.05$ or $\mathrm{P}<0.01$; Figs. $1 \mathrm{~A}$ and 2$)$. The $\mathrm{mRNA}$ and protein expression levels of ASC and caspase-1 were significantly reduced in the $\mathrm{SCI}+\mathrm{HBO}$ group compared with those in the SCI group 3 and 7 days after surgery $(\mathrm{P}<0.05$; Figs. $1 \mathrm{~B}$ and C, 3 and 4).

HBOT downregulates the SCI-induced release of $I L-1 \beta$. The levels of IL-1 $\beta$ in the injured spinal cord tissue was significantly increased compared with those in the $\mathrm{SH}$ and $\mathrm{SH}+\mathrm{HBO}$ groups at any time points and peaked 1 day after surgery. Compared with the SCI group, the IL-1 $\beta$ level in the SCI+HBO group was significantly decreased 3 and 7 days after surgery $(\mathrm{P}<0.05 ; \mathrm{P}<0.01$; Table III).

\section{Discussion}

The NALP3 inflammasome is a well-characterized inflammasome consisting of NALP3, ASC and caspase-1. NALP3 is a member of the NOD-like receptor family, which has the ability to sense pathogen products in the cytoplasm (16) and elicit an immune response. The NALP3 protein recruits the adaptor molecule ASC via an N-terminal protein-protein interaction in the pyrin domain. ASC activates caspase-1 via its $\mathrm{C}$-terminal domain, subsequently leading to the release of mature IL-1 $\beta(17,18)$.

At the stage of secondary damage following SCI, formation of oxygen radicals and cell membrane lipid peroxidation are observed $(19,20)$. Overproduction of ROS causes irreversible damage to neurons, including organelles and membranes. However, ROS-mediated intracellular signaling pathways following SCI remain to be fully elucidated. ROS are implicated in the activation of the NALP3 inflammasome (21). Kauppinen et al (22) examined the ability of ROS to activate inflammasome signaling in retinal pigment epithelium (RPE) cells and demonstrated that 4-hydroxy-2-nonenal, a highly reactive end-product of the lipid peroxidation reaction, significantly increased the mRNA level of NALP3. This study provided 
A

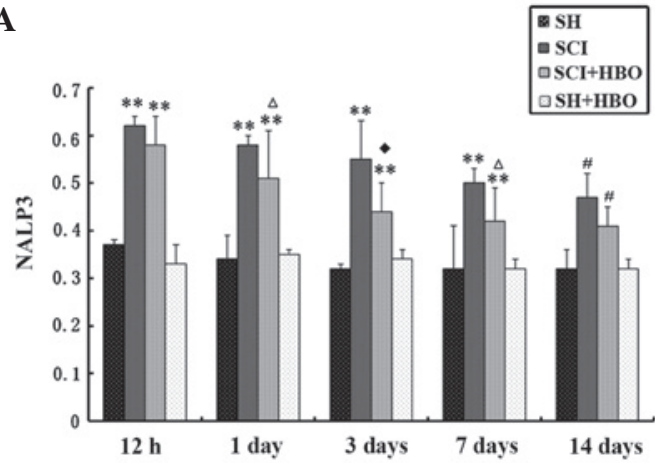

B

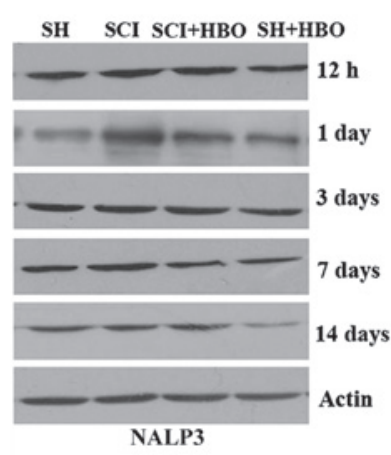

Figure 2. (A) Quantification analysis of NALP3 and (B) immunoblot of NALP3 in the spinal cords of all groups of rats. Data are presented as the mean \pm standard deviation. ${ }^{* *} \mathrm{P}<0.01$ for $\mathrm{SCI}$ and $\mathrm{SCI}+\mathrm{HBO}$ groups, vs. $\mathrm{SH}$ and $\mathrm{SH}+\mathrm{HBO}$ groups; ${ }^{*} \mathrm{P}<0.05$ for $\mathrm{SCI}$ and $\mathrm{SCI}+\mathrm{HBO}$ groups, vs. $\mathrm{SH}$ and $\mathrm{SH}+\mathrm{HBO}$ groups; ${ }^{\bullet} \mathrm{P}<0.01$ and ${ }^{\triangle} \mathrm{P}<0.05$ for SCI+HBO group, vs. SCI group. NALP3, NACHT domain-, leucine-rich-repeat- and pyrin domain-containing protein 3; SCI, spinal cord injury group; HBO, hyperbaric oxygen treatment; $\mathrm{SH}$, sham-operated.

A

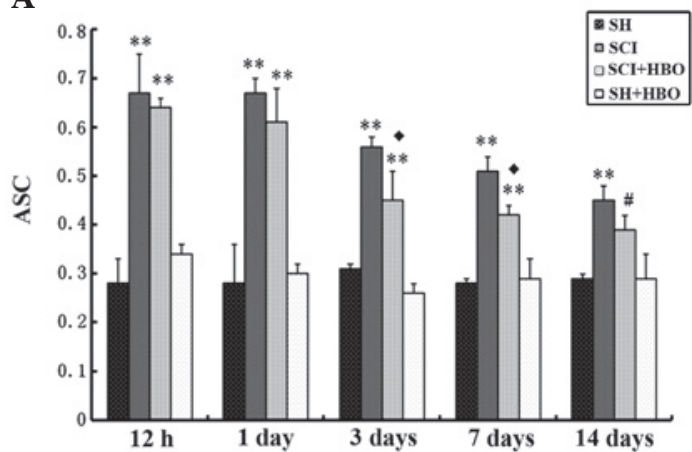

B

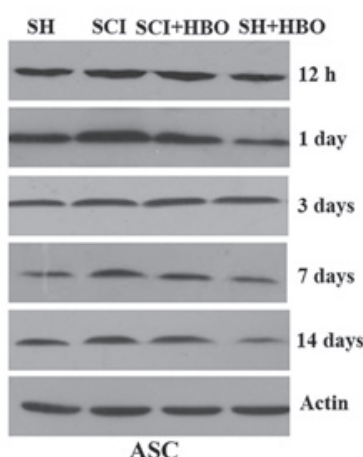

Figure 3. (A) Quantification analysis of ASC and (B) immunoblot of ASC in the spinal cords of all groups of rats. Data are presented as the mean \pm standard deviation. ${ }^{* *} \mathrm{P}<0.01$ for $\mathrm{SCI}$ and $\mathrm{SCI}+\mathrm{HBO}$ groups, vs. $\mathrm{SH}$ and $\mathrm{SH}+\mathrm{HBO}$ groups; ${ }^{*} \mathrm{P}<0.05$ for $\mathrm{SCI}$ and $\mathrm{SCI}+\mathrm{HBO}$ groups, vs. SH and $\mathrm{SH}+\mathrm{HBO}$ groups; ${ }^{\bullet} \mathrm{P}<0.01$ and ${ }^{\Delta} \mathrm{P}<0.05$ for SCI+HBO group, vs. SCI group. ASC, apoptosis-associated speck-like proteins; SCI, spinal cord injury; HBO, hyperbaric oxygen treatment; $\mathrm{SH}$, sham-operated.

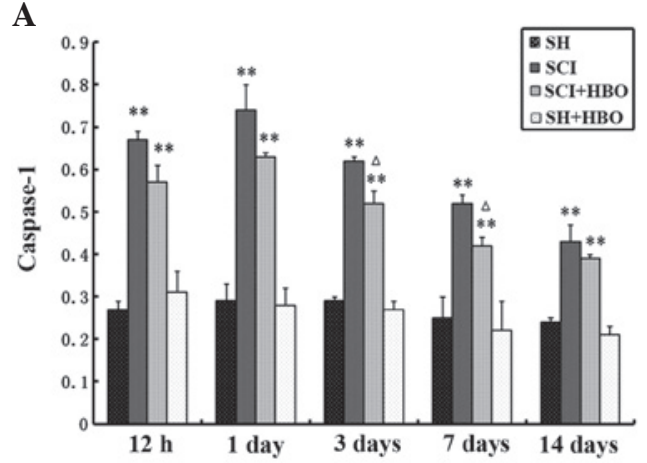

B



Figure 4. (A) Quantification analysis of caspase-1 and (B) immunoblot of caspase-1 in the spinal cords of all groups of rats Data are presented as the mean \pm standard deviation. ${ }^{* * *} \mathrm{P}<0.01$ for $\mathrm{SCI}$ and $\mathrm{SCI}+\mathrm{HBO}$ groups, vs. $\mathrm{SH}$ and $\mathrm{SH}+\mathrm{HBO}$ groups; and ${ }^{\triangle} \mathrm{P}<0.05$ for $\mathrm{SCI}+\mathrm{HBO}$ group, vs. SCI group. SCI, spinal cord injury; HBO, hyperbaric oxygen treatment; $\mathrm{SH}$, sham-operated.

evidence that oxidative stress can activate NALP3 inflammasome signaling in RPE cells, which are important in the pathogenesis of age-related macular degeneration. Western blotting and RT-qPCR analysis in the current study demonstrated that the expression levels of the inflammasome components, including NALP3, ASC and caspase-1, were up-regulated in the SCI groups compared with the $\mathrm{SH}$ and $\mathrm{SH}+\mathrm{HBO}$ groups and levels peaked $12 \mathrm{~h}$ and 1 day after surgery respectively. The NALP3 protein is a core component of the inflammasome.
The present study revealed that the mRNA levels of NALP3 in the SCI groups were 2.2-fold higher compared with the $\mathrm{SH}$ groups. The levels of the adaptor protein ASC were moderately induced during the process. The mRNA levels in the SCI groups were 2.7-fold higher compared with the SH and $\mathrm{SH}+\mathrm{HBO}$ groups. Pro-caspase-1 is an substrate-specific protease and is recruited by ASC via its C-terminal domain and cleaved to form active caspase-1. Levels of ASC-induced cleavage of pro-caspase-1 were measured in the present study. 
The mRNA levels of caspase-1 were 3.1-fold higher in the SCI group compared with the $\mathrm{SH}$ and $\mathrm{SH}+\mathrm{HBO}$ groups. Juliana et al suggested that NALP3 requires post-translational modifications prior to the inflammasome activation (23). Among the modifications, de-ubiquitylation of NALP3 is critical for its activation, possibly in a mitochondrial ROS-dependent manner (24-26). Based on previous studies, the present study hypothesized that following SCI, overproduction of ROS promotes NALP3 de-ubiquitylation, facilitating its activation and enhancing its ability to interact with ASC and caspase-1. The present study demonstrated that simultaneous upregulation of NALP3, caspase-1 and ASC occurs following SCI, suggesting that the ROS generated in the process of second injury induces the formation of the functional NALP3 inflammasome.

HBOT has been demonstrated to ameliorate the hypoxic state at the site of SCI by decreasing MDA levels and increasing levels of SOD, GSH, and CAT $(27,28)$. These studies indicated that HBOT reduced the formation of ROS to protect the injured neurons of the spinal cord during this stage of trauma $(29,30)$. In the present study, the mRNA and protein expression levels of NALP3 in the SCI+HBO group were significantly reduced compared with those in the SCI group 1, 3 and 7 days after surgery. The mRNA and protein expression levels of ASC and caspase- 1 were significantly reduced in the $\mathrm{SCI}+\mathrm{HBO}$ group compared with those in the SCI group 3 and 7 days after surgery. A study by Rubartelli suggested that cellular oxidation and its own anti-oxidant reactions may be involved in modulating NALP3 activation (31). Therefore, the present study hypothesized that HBOT hypersaturated circulating plasma with dissolved oxygen, increasing the oxygen tension around the injured spinal cord tissue and upregulating antioxidant ability of tissue to counter ROS production. Inhibition of ROS is hypothesized to prevent the activation of the NALP3 inflammasome.

IL-1 $\beta$ is important in the pathogenesis and progression of secondary injury in SCI. In vitro, expression levels of IL-1 $\beta$ were increased following SCI, with the mRNA and protein levels peaking $1 \mathrm{~h}$ and $8 \mathrm{~h}$ after injury, respectively and levels remained elevated for at least $72 \mathrm{~h}(32,33)$. The pro-inflammatory cytokine IL-1 $\beta$ is central in initiating an inflammatory response. Pro-IL-1 $\beta$ can be produced through Toll-like receptors (TLRs) and NF- $\mathrm{NB}$ signaling, and remain as inactive intracellular precursors until they are activated (34). Caspase-1 cleaves a 116 amino acid region from the $\mathrm{N}$-terminus of pro-IL-1 $\beta$ to convert it into active IL-1 $\beta$. Kool et al demonstrated that aluminium triggers macrophages to release mature IL-1 $\beta$ in a NALP3- and ASC-dependent manner (35). In the present study, the levels of IL-1 $\beta$ in spinal cord tissue were analyzed using ELISA. IL-1 $\beta$ was significantly increased and peaked $24 \mathrm{~h}$ after surgery. Notably, HBOT significantly decreased the levels of IL-1 $\beta 3$ and 7 days after surgery compared with the SCI groups. These data suggested that the increased level of IL-1 $\beta$ aligned with the increased level of caspase- 1 following SCI. Notably, HBOT compromised expression of caspase-1 and IL-1 $\beta$. Yang et al (36) indicated that HBOT could reduce the secondary injury of SCI via down-regulating the expression of $\mathrm{NF}_{-\kappa \mathrm{B}}$. These data suggested that HBOT attenuated the secondary injury of SCI due to the inhibition of IL-1 $\beta$ transcription and inhibition of the NALP3 inflammasome pathway, which activates IL-1 $\beta$.
In order to evaluate the recovery of motor function, BBB scores were measured. The BBB scores were significantly lower in the SCI and SCI+HBO groups compared with the $\mathrm{SH}$ group. However, the BBB score was significantly increased in the SCI+HBO groups compared with the SCI groups on the 7 th and 14th days after surgery. The results of the BBB scores suggested that HBOT preserved the injured spinal cord tissue, promoting neurological recovery following SCI.

In conclusion, the present study demonstrated that HBOT prevents spinal cord injury by inhibiting the activation of the NALP3 inflammasome, including all the components of this pathway. The results demonstrated that HBOT is an effective approach to relieve secondary injury in the early stages of SCI.

\section{Acknowledgements}

The present study was supported by a grant from the Program of International S\&T Cooperation (no. 2012DFA31240).

\section{References}

1. Dumont RJ, Okonkwo DO, Verma S, Hurlbert RJ, Boulos PT, Ellegala DB and Dumont AS: Acute spinal cord injury, part I: Pathophysiologic mechanisms. Clin Neuropharmacol 24: 254-264, 2001.

2. Kwon BK, Tetzlaff W, Grauer JN, Beiner J and Vaccaro AR: Pathophysiology and pharmacologic treatment of acute spinal cord injury. Spine J 4: 451-464, 2004.

3. Hall ED and Springer JE: Neuroprotection and acute spinal cord injury: a reappraisal. NeuroRx 1: 80-100, 2004.

4. Beck KD, Nguyen HX, Galvan MD, Salazar DL, Woodruff TM and Anderson AJ: Quantitative analysis of cellular inflammation after traumatic spinal cord injury: evidence for a multiphasic inflammatory response in the acute to chronic environment. Brain 133: 433-447, 2010.

5. Duz B, Kaplan M, Bilgic S, et al: Does hypothermic treatment provide an advantage after spinal cord injury until surgery? An experimental study. Neurochem Res 34: 407-410,2009.

6. Diaz-Ruiza A, Maldonadoc PD, Mendez-Armenta M, et al: Activation of heme oxygenase recovers motor function after spinal cord injury in rats. Neurosci Lett 556: 26-31, 2013.

7. Maggio DM, Chatzipanteli K, Masters N, Patel SP, Dietrich WD and Pearse DD: Acute molecular perturbation of inducible nitric oxide synthase with an antisense approach enhances neuronal preservation and functional recovery after contusive spinal cord injury. J Neurotrauma 29: 2244-2249, 2012.

8. Kubeck JP, Merola A, Mathur S, et al: End organ effects of high-dose human equivalent methylpredniso-lone in a spinal cord injury rat model. Spine 31: 257-261,2006.

9. Kahraman S, Düz B, Kayali H, et al: Effects of methylprednisolone and hyperbaric oxygen on oxidative status after experimental spinal cord injury: a comparative study in rats. Neurochem Res 32: 1547-1551, 2007

10. Gamache FW Jr, Myers RA, Ducker TB, et al: The clinical application of hyperbaric oxygen therapy in spinal cord injury: a preliminary report. Surg Neurol 15: 85-87, 1981.

11. Kindwall EP, Gottlieb LJ and Larson DL: Hyperbaric oxygen therapy in plasticsurgery: a review article. Plast Reconstr Surg 88: 898-908, 1991.

12. Petrilli V, Dostert C, Muruve DA, et al: The inflammasome: a danger sensing complex triggering innate immunity. Current Opin Immunol 19: 615-622, 2007.

13. Lamkanfi $\mathbf{M}$ and Dixit $\mathrm{M}$ : Inflammasomes: guardians of cytosolic sanctity. Immunol. Rev 227: 95-105, 2009.

14. Villegas LR, Field C, Kluck D, et al: Superoxide dismutase mimetic attenuates hypoxia-induced pulmonary vascular remodeling, ECM hyaluronan expression, and NALP3-mediated inflammation. Free Radical Bio Med 10:364,2011.

15. Basso DM, Beattie MS and Bresnahan JC: Graded histological and locomotor outcomes after spinal cord contusion using the NYU weight drop device versus transaction. Exp Neurol 139: 244-256, 1996 
16. Martinon F and Tschopp J: NLRs join TLRs as innate sensors of pathogens. Trends Immunol 26: 447-454, 2005.

17. Aqostin L, Martinon F, Burns K, et al: NALP3 forms an L-1beta-processing inflammasome with increased activity in Muckle-Wells autoinflammatory disorder. Immunity 20 319-325, 2004.

18. Chen M, Wang H, Chen W, et al: Regulation of adaptive immunity by the NLRP3 inflammasome. Int Immunopharmacol 11: 549-555, 2011.

19. Bao F, Dekaban GA and Weaver LC: Anti-CD11d antibody treatment reduces free radical formation and cell death in the injured spinal cord of rats. J Neurochem 94: 1361-1373, 2005.

20. Hachmeister JE, Valluru L, Bao F and Liu D: Mn (III) tetrakis (4-benzoic acid) porphyrin administered into the intrathecal space reduces oxidative damage and neuron death after spinal cord injury: a comparison with methylprednisolone. J Neurotrauma 23: 1766-1778, 2006.

21. Allen IC, Scull M A, Moore CB, et al: The NLRP3 inflammasome mediates in vivo innate immunity to influenza A virus through recognition of viral RNA. Immunity 30: 556-565, 2009

22. Kauppinen A, Niskanen H, Suuronen T, Kinnunen K, Salminen A and Kaarniranta K: Oxidative stress activates NLRP3 inflammasomes in ARPE-19 cells - implications for age-related macular degeneration (AMD). Immunol Lett 147: 29-33, 2012.

23. Juliana C, Fernandes-Alnemri T, Kang S, Farias A, Qin F and Alnemri ES: Non-transcriptional priming and deubiquitination regulate NLRP3 inflammasome activation. J Biol Chem 287: 36617-36622, 2012.

24. Menu P and Vince JE: The NLRP3 inflammasome in health and disease: the good, the bad and the ugly. Clin Exp Immunol 166 $1-15,2011$.

25. Lawlor KE and Vince JE: Ambiguities in NLRP3 in flammasome regulation: is there a role for mitochondria? Biochim Biophys Acta 10: 08-14, 2013.

26. Vince IE, Wong WW, Gentle I, et al: Inhibitor of apoptosis proteins limit RIP3 kinase-dependent interleukin-1 activation. Immunity 36: 215-227, 2012.
27. Yaman O, Yaman B, Aydin F, Var A and Temiz C: Hyperbaric oxygen treatment in the experimental spinal cord injury model. Spine J 14: 2184-2194, 2014.

28. Ishihara H, Kanamori M, Kawaguchi Y, Osada R, Ohmori K and Matsui H: Prediction of neurologic outcome in patients with spinal cord injury by using hyperbaric oxygen therapy. J Orthop Sci 6: 385-389, 2001

29. Topuz K, Colak A, Cemil B, et al: Combined hyperbaric oxygen and hypothermia treatment on oxidative stress parameters after spinal cord injury: an experimental study. Arch Med Res 41: 506-512, 2010

30. Yu Y, Matsuyama Y, Yanase M, et al: Effects of hyperbaric oxygen on GDNF expression and apoptosis in spinal cord injury. Neuroreport 15: 2369-2373, 2004.

31. Rubartelli A: Redox control of NLRP3 inflammasome activation in health and disease. J Leukoc. Biol 92: 951-958, 2012.

32. Liu S, Xu GY, Johnson KM, et al: Regulation of interleukin-1beta by the interleukin-1 receptor antagonist in the glutamate-injured spinal cord: endogenous neuroprotection. Brain Res 1231: 63-74, 2008.

33. Sung CS, Wen ZH, Chang WK, et al: Intrathecal interleukin-1beta administration induces thermal hyperalgesia by activating inducible nitric oxide synthase expression in the rat spinal cord. Brain Res 1015: 145-153, 2004.

34. Martinon F, Burns K and Tschopp J: The inflammasome: a molecular platform triggering activation of inflammatory caspases and processing of pro IL-beta. Mol Cell 10: 417-426, 2002.

35. Kool, M., Pétrilli V, De Smedt T, et al: Cutting edge: alum adjuvant stimulates inflammatory dendritic cells through activation of the NALP3 inflammasome. J Immunol 181 3755-3759, 2008

36. Yang J, Liu X, Zhou Y, Wang G, Gao C, Su Q: Hyperbaric oxygen alleviates experimental (spinal cord) injury by downregulating HMGB1/NF-kB expression. Spine 38: 1641-1648, 2013. 